\title{
Fatal acute melioidosis in a tourist returning from Martinique Island, November 2010
}

L Gétaz (laurent.getaz@hcuge.ch)1, M Abbas², L Loutan¹, J Schrenzel ${ }^{3,4}$, A Iten5, F Simon ${ }^{6}$, A Decosterd7, R Studer ${ }^{3}$, P Sudre ${ }^{8}$,

Y Michel ${ }^{1}$, P Merlani ${ }^{7}, \mathrm{~S}$ Emonet ${ }^{3,4}$

1. Division of Humanitarian and International Medicine, Department of Community Medicine and Primary Care, Geneva University Hospitals, and Faculty of Medicine, University of Geneva, Switzerland

2. Service of General Internal Medicine, Department of Internal Medicine, Geneva University Hospitals, Switzerland

3. Bacteriology Laboratory, Department of Genetic Medicine and Laboratories, Geneva University Hospitals, Switzerland

4. Service of Infectious Diseases, Department of Internal Medicine, Geneva University Hospitals, Switzerland

5. Infection Control Program, Geneva University Hospitals, Switzerland

6. Infectious Diseases and Tropical Medicine Unit, Laveran Military Teaching Hospital, Marseille, France

7. Service of Intensive Care, Department of Anaesthesiology, Pharmacology and Intensive Care, Geneva University Hospitals and University of Geneva, Switzerland.

8. General directorate of health, Department of regional affairs, economy and health, Canton of Geneva, Switzerland

Citation style for this article:

Gétaz L, Abbas M, Loutan L, Schrenzel J, Iten A, Simon F, Decosterd A, Studer R, Sudre P, Michel Y, Merlani P, Emonet S. Fatal acute melioidosis in a tourist returning from Martinique Island, November 2010. Euro Surveill. 2011;16(1):pii=19758. Available online: http://www.eurosurveillance.org/ViewArticle.aspx?Articleld=19758

Article published on 6 January 2011

We report the fatal case of acute melioidosis in a patient returning from Martinique with fever in November 2010. Gram-negative rods were isolated from a blood culture and Burkholderia pseudomallei identified within 24 hours after first medical contact. The patient died two days after admission to hospital despite intravenous therapy with high doses of imipenem/cilastatin and intensive care. Clinicians seeing travellers returning from the subtropics or tropics with severe pneumonia or septicaemia should consider the possibility of acute melioidosis.

\section{Introduction}

Melioidosis is an infectious disease of public health importance that is endemic in southeast Asia and northern Australia. However, cases are increasingly recognised in other parts of the world, including Africa, Latin America, and the Middle East. In the Caribbean, few cases of melioidosis have been reported: in Puerto Rico during flood waters, in Guadeloupe and a single case 15 years ago from an inhabitant of Martinique [1-3]. Melioidosis is occasionally reported in travellers returning from Asia, including in three Finnish tourists after the tsunami of 2004 [4-10]. Melioidosis in travellers from other regions is rarely reported: a tourist who presented with acute fatal septicaemic pneumonia due to melioidosis after a visit to Brazil was diagnosed in the Netherlands [11].

Burkholderia pseudomallei, the causative organism, is a saprophyte living in soil and surface water [12]. Although the exact mode of transmission of this disease remains unknown, percutaneous inoculation and especially exposure of non-intact skin to contaminated soil or surface water is mostly reported in the literature [13]. Other modes of transmission include inhalation of contaminated water or soil dust, laboratory hazards and, rarely, person-to-person through contact with blood or body fluids [14].

The clinical presentation and the spectrum of severity range from fulminant sepsis to chronic disease. The most common manifestations are pneumonia, sepsis and intra-abdominal suppuration. Other common manifestations include parotitis, abscesses of the prostatic gland and of other organs as well as encephalomyelitis [12]. The incubation period can be as short as one day; however, years may lapse between presumed exposure and appearance of the chronic clinical disease [14]. Despite optimal antibiotic treatment, acute melioidosis is still associated with significant mortality, between $30 \%$ and $50 \%$ in cases with severe sepsis [12].

\section{Case report}

A healthy man in his 30 s living in Switzerland visited the east coast of Martinique 10 days in November 2010. He suffered from a few episodes of mild watery diarrhoea for some days during this period and became febrile on the last day of his stay, with chills, headache, abdominal cramps and lower back pain, without articular or neurological complaints. He did not seek medical advice until two days later, following his return home, when he was admitted to the Geneva University Hospitals on 25 November.

Biological findings showed systemic inflammatory response syndrome with a leukocyte count of $10 \times 10^{9} / \mathrm{L}, 97 \%$ of neutrophils and $39 \%$ band forms (immature white blood cells), elevated C-reactive protein $(315 \mathrm{mg} / \mathrm{L})$ and elevated transaminases: aspartate aminotransferase $257 \mathrm{IU} / \mathrm{L}$ (norm: 14-50) and alanine aminotransferase $128 \mathrm{IU} / \mathrm{L}$ (norm: 12-50). Gramnegative rods grew from blood cultures after 24 hours of incubation. Mass spectrometry (MS) was performed according to standard practise in our laboratory [15]. 
Burkholderia sp. was identified on a very thin layer of bacteria grown on blood agar two hours after subculture. The identification score from the MS was 1.8 , corresponding to secure identification at the gender level (according to the manufacturer). Initially medical staff suspected typhoid fever, and the patient was treated with ceftriaxone. Once the first bacteriological results were available, this was changed to highdose imipenem-cilastatin therapy. The presence of Burkholderia sp. in the blood of a previously healthy patient returning from subtropical area, and developing rapidly progressive respiratory failure led to the presumption of melioidosis. MS was performed again the same day, 26 November, on a single well-grown colony and a score $>2$ of $B$. thailandensis was identified. Hereafter, sequencing of the $16 \mathrm{~S}$ rDNA confirmed the presence of $B$. pseudomallei in all blood cultures.

Despite the absence of respiratory symptoms and a normal initial chest X-ray on 25 November, the patient developed respiratory failure the following day, due to severe bilateral abscess-forming pneumonia. An X-ray and a computed tomography at 36 hours after admission showed bilateral infiltrates with nodular lesions probably corresponding to micro-abscesses. Dysuria with pyuria appeared 36 hours after admission. The patient died on the second day of admission from septic shock with multi-organ failure and acute respiratory distress syndrome, in spite of additional therapy with granulocyte colony-stimulating factor (G-CSF), lowdose steroids and intensive care including extracorporeal membrane oxygenation.

\section{Discussion}

This is the first reported case, to our knowledge, of melioidosis in a traveller returning from Martinique. Clusters of cases are known to occur mainly in hyperendemic countries. But following natural disasters (storms, typhoons, tsunamis) they are also seen in areas of lower endemicity [16-19]. At the end of October 2010, Martinique was affected by hurricane Tomas. Two weeks later, following three days of heavy rain, the areas visited by our patient experienced flooding. Severe melioidosis is strongly correlated with heavy rain, considered an independent risk factor for a pneumonic presentation, septic shock, and death [19].

The majority of cases are seen in patients with a predisposing medical condition such as diabetes, cirrhosis, alcoholism or renal failure. One third of infections affect healthy subjects, as in the case of our patient [14]. As soon as the diagnosis of melioidosis was suspected, more details of his medical history were recorded: risk factors such as walking in the mud in a forest and across recently flooded areas with multiple scratched mosquito bites on his legs were identified. Information about suspected contaminated areas in Martinique where the patient had walked in the mud and names of other tourists exposed to the mud (the patient participated in a surf camp with several French tourists) was communicated within two days following the patient's hospital admission to the local clinicians in Martinique, international travel medicine networks (EuroTravNet, TropNetEurop and GeoSentinel), as well as to Swiss and French national health authorities. Following our informing the local hospitals in Martinique, two additional cases were suspected and antibiotic therapy immediately adapted. Local authorities will investigate areas considered as suspicious for potential transmission of the pathogen.

Rapid diagnostic methods are critical as the clinical presentation of melioidosis is non-specific and treatment requires specific antibiotics. Conventional techniques, including Gram staining and culture remain the mainstay of diagnosis for melioidosis, but require 24 hours for isolation and an additional 48 hours for species identification [12]. In our case, MS initially detected $B$. thailandensis. $B$. thailandensis cannot be reliably distinguished from $B$. mallei or B.pseudomalle $i$ by MS. $B$ thailandensis and $B$. pseudomallei are very closely related species with similar susceptibility to antibiotics, but with a completely different clinical spectrum. Infections caused by the former are usually asymptomatic or cause mild disease, whereas infections caused by the latter are always highly pathogenic and cause acute or chronic manifestations [20].

B. pseudomallei has been classified as a potential bioterrorism agent and should be handled with biosafety level 3 in laboratories. Few cases of laboratoryacquired melioidosis have been reported. The level of risk exposure in the laboratory determines the need for post-exposure prophylaxis [21]. In our case, two laboratory assistants were exposed to aerosols of $B$. pseudomallei cultures and another two were exposed to patient specimens (urine and faeces). Exposure to the latter was considered low-risk and post-exposure prophylaxis (PEP) was stopped after 72 hours (culture negative). Exposure to positive culture aerosols was considered to be high-risk and PEP using the 3-weeks co-trimoxazole scheme proposed by Peacock et al. was performed [21].

Matrix-assisted laser desorption/ionisation timeof-flight (MALDI-TOF) MS provides rapid identification of the agent of melioidosis, with the potential to improve the prognosis of fulminant melioidosis $[15,22]$. However, the availability of this new technology in developing countries is still limited. Identification techniques such as indirect haemagglutination and other serological tests have limited utility for clinical case management [13]. The gold standard for identification still remains $16 \mathrm{~S}$ rDNA sequencing, but this is timeconsuming and costly.

All cases of melioidosis, even mild disease, should be treated with initial intensive therapy (two to four weeks of intravenous antibiotics). Recommended intravenous therapy is ceftazidime, imipenem or meropenem [13]. G-CSF is sometimes proposed as adjuvant therapy and low-dose steroids are suggested as beneficial in 
refractory shock [12]. Intensive therapy should always be followed by at least 12 weeks of oral eradication therapy, using typically trimethoprim-sulfamethoxazole with doxycycline [12].

\section{Conclusion}

As clusters of melioidosis are known to occur, local medical authorities and international travel medicine networks should be informed quickly when a case is diagnosed. This could be particularly critical when the contaminated area is a touristic location where the disease is rarely reported. Prompt reporting facilitates the investigation of possible additional cases, the intensification of security measures in laboratories, and the realisation of a field study to analyse the soil in suspect areas.

\section{Acknowledgements}

The authors are grateful to $\mathrm{Dr}$ Claude Olive, $\mathrm{Dr}$ Rafaelle Theodose (Fort de France Teaching Hospital, Martinique) and Dr Myrta Vesanes (Le Marin Hospital, Martinique) for their collaboration, making additional case finding possible. We also thank the staff of the vacation centre in Le Vauclin and $\mathrm{Dr}$ Michel Jacquin for their help in contact tracing. We thank Dr Philippe Parola, coordinator of EuroTravNet, for his helpful advice and Dr Francesca Savioli for proofreading the manuscript.

\section{References}

1. Christenson B, Fuxench Z, Morales JA, Suárez-Villamil RA, Souchet LM. Severe community-acquired pneumonia and sepsis caused by Burkholderia pseudomallei associated with flooding in Puerto Rico. Bol Asoc Med P R. 2003 Nov-Dec;95(6):17-20.

2. Olive C, Loetitia G, Desbois N, Roche B, Jouannelle J, Dodin A. Septic pyemic form of human melioidosis: a first case in the French Antilles. Presse Med. 1995 Sep 23;24(27):1270.

3. Pérez JM, Petiot A, Adjidé C, Gerry F, Goursaud R, Juminer B. First case report of melioidosis in Guadeloupe, a French West Indies archipelago. Clin Infect Dis. 1997 Jul;25(1):164-5.

4. Badran S, Pedersen TI, Roed C, Lunding S, Birk N, Vestergaard $\mathrm{H}$ et al. Imported melioidosis in Danish travellers: a diagnostic challenge. Scand J Infect Dis. 2010 Jul;42(6-7):445-9.

5. Ezzedine K, Heenen M, Malvy D. Imported cutaneous melioidosis in traveler, Belgium. Emerg Infect Dis. 2007 Jun;13(6):946-7.

6. Ezzedine K, Malvy D, Steels E, De Dobbeeler G, Struelens M, Jacobs F et al. Imported melioidosis with an isolated cutaneous presentation in a 90-year-old traveller from Bangladesh. Bull Soc Pathol Exot. 2007 Feb;100(1):22-5.

7. Yazdanpanah Y, Lemaire X, Senneville E, Delcey V, Viget $\mathrm{N}$, Mouton $\mathrm{Y}$ et al. Melioidotic osteomyelitis of the femur occurring in a traveler. J Travel Med. 2002 Jan-Feb;9(1):53-4.

8. Maccanti O, Pardelli R, Tonziello A, Gracci G, Vivaldi I, Bolognesi L et al. Melioidosis in a traveller from Thailand: case report. J Chemother. 2004 Aug;16(4):404-7.

9. Nieminen T, Vaara M. Burkholderia pseudomallei infections in Finnish tourists injured by the December 2004 tsunami in Thailand. Euro Surveill. 2005;10(9):pii=2656. Available from: http://www.eurosurveillance.org/ViewArticle. aspx?Articleld $=2656$

10. Dance DA, Smith MD, Aucken HM, Pitt TL. Imported melioidosis in England and Wales. Lancet. 1999 Jan 16;353(9148):208.

11. Aardema H, Luijnenburg EM, Salm EF, Bijlmer HA, Visser CE, Van't Wout JW. Changing epidemiology of melioidosis? A case of acute pulmonary melioidosis with fatal outcome imported from Brazil. Epidemiol Infect. 2005 Oct;133(5):871-5.

12. Cheng AC. Melioidosis: advances in diagnosis and treatment. Curr Opin Infect Dis. 2010 Dec;23(6):554-9

13. Currie B. Treatment and prognosis of melioidosis / Epidemiology, pathogenesis, clinical manifestations, and diagnosis of melioidosis. In: UpToDate, Basow, DS (Ed), UpToDate, Waltham, MA, 2010.

14. Heymann D. Melioidosis. In: Control of communicable disease. 18th edition. Washington: American Public Health Association; 2004.

15. Emonet S, Shah HN, Cherkaoui A, Schrenzel J. Application and use of various mass spectrometry methods in clinical microbiology. Clin Microbiol Infect. 2010 Nov;16(11):1604-13.

16. Peacock SJ. Melioidosis. Curr Opin Infect Dis. 2006 Oct;19(5):421-8.

17. Inglis TJ, Rolim DB, Sousa Ade Q. Melioidosis in the Americas. Am J Trop Med Hyg. 2006 Nov;75(5):947-54.

18. Ko WC, Cheung BM, Tang HJ, Shih HI, Lau YJ, Wang LR, et al. Melioidosis outbreak after typhoon, southern Taiwan. Emerg Infect Dis. 2007 Jun;13(6):896-8.

19. Currie BJ, Dance DA, Cheng AC.The global distribution of Burkholderia pseudomallei and melioidosis: an update. Trans $\mathrm{R}$ Soc Trop Med Hyg. 2008 Dec;102 Suppl 1:S1-4.

20. Glass MB, Gee JE, Steigerwalt AG, Cavuoti D, Barton T, Hardy RD, et al. Pneumonia and septicemia caused by Burkholderia thailandensis in the United States. J Clin Microbiol. 2006 Dec: 44(12): 4601-2.

21. Peacock SI, Schweizer HP, Dance DA, Smith TL, Gee JE, Wuthiekanun $\mathrm{V}$ et al. Management of accidental laboratory exposure to Burkholderia pseudomallei and B. mallei. Emerg Infect Dis. 2008 Jul;14(7):e2.

22. Cherkaoui A, Hibbs J, Emonet S, Tangomo M, Girard M, Francois $P$ et al., Comparison of two matrix-assisted laser desorption ionization-time of flight mass spectrometry methods with conventional phenotypic identification for routine identification of bacteria to the species level. J Clin Microbiol. 2010 Apr;48(4):1169-75. 
